\title{
Gestational diabetes with diabetes and prediabetes risks: a large observational study
}

\author{
Yun Shen 1,2,*, Peng Wang ${ }^{3, *}$, Leishen Wang ${ }^{3}$, Shuang Zhang ${ }^{3}$, Huikun $\mathrm{Liu}^{3}$, Weiqin $\mathrm{Li}^{3}$, Nan $\mathrm{Li}^{3}$, Wei $\mathrm{Li}^{3}$, \\ Junhong Leng ${ }^{3}$, Jing Wang ${ }^{3}$, Huiguang Tian ${ }^{3}$, Cuilin Zhang ${ }^{4}$, Jaakko Tuomilehto5,6, Xilin Yang ${ }^{7}$, Zhijie Yu ${ }^{8}$ and \\ Gang Hu' ${ }^{1}$
}

${ }^{1}$ Pennington Biomedical Research Center, Baton Rouge, Louisiana, USA, 2Department of Endocrinology and Metabolism, Shanghai Jiao Tong University Affiliated Six People's Hospital, Shanghai, China, ${ }^{3}$ Tianjin Women's and Children's Health Center, Tianjin, China, ${ }^{4}$ Division of Intramural Population Health Research, Eunice Kennedy Shriver National Institute of Child Health and Human Development, National Institutes of Health, Bethesda, Maryland, USA, ${ }^{5}$ Dasman Diabetes Institute, Dasman, Kuwait, ${ }^{6}$ Department of Public Health, University of Helsinki, Helsinki, Finland, 'Department of Epidemiology, School of Public Health, Tianjin Medical University, Tianjin, China, and ${ }^{8}$ Population Cancer Research program, Dalhousie University, Halifax, Nova Scotia, Canada

*(Y Shen and P Wang contributed equally to this work)

Correspondence should be addressed to $\mathrm{G} \mathrm{Hu}$

Email gang.hu@pbrc.edu

\begin{abstract}
Aims: To compare risks of early postpartum diabetes and prediabetes in Chinese women with and without gestational diabetes mellitus (GDM) during pregnancy.

Subjects and methods: Tianjin GDM observational study included 1263 women with a history of GDM and 705 women without GDM who participated in the urban GDM universal screening survey by using World Health Organization's criteria. Postpartum diabetes and prediabetes were identified after a standard oral glucose tolerance test. Cox proportional hazards regression was used to assess risks of postpartum diabetes and prediabetes between women with and without GDM.

Results: During a mean follow-up of 3.53 years postpartum, 90 incident cases of diabetes and 599 incident cases of prediabetes were identified. Multivariable-adjusted hazard ratios among women with prior GDM, compared with those without it, were 76.1 (95\% Cl: 23.6-246) for diabetes and 25.4 (95\% Cl: 18.2-35.3) for prediabetes. When the mean follow-up extended to 4.40 years, 121 diabetes and 616 prediabetes cases were identified. Women with prior GDM had a 13.0-fold multivariable-adjusted risk (95\% Cl: 5.54-30.6) for diabetes and 2.15-fold risk (95\% Cl: 1.762.62) for prediabetes compared with women without GDM. The positive associations between GDM and the risks of postpartum diabetes and prediabetes were significant and persistent when stratified by younger and older than 30 years at delivery and normal weight and overweight participants.

Conclusions: The present study indicated that women with prior GDM had significantly increased risks for postpartum diabetes and prediabetes, with the highest risk at the first 3-4 years after delivery, compared with those without GDM.
\end{abstract}

\section{Introduction}

The prevalence of diabetes and prediabetes were $10.9 \%$ and $35.7 \%$ in 2013 in China respectively (1). The prevalence of gestational diabetes mellitus (GDM) has increased from
European Journal of

Endocrinology

(2018) 179, 51-58 www.eje-online.org

https://doi.org/10.1530/EJE-18-0130
C) 2018 European Society of Endocrinology Printed in Great Britain
$2.4 \%$ in 1999 to $8.1 \%$ in 2012 in urban China $(2,3)$, now close to the US level ( 7\%) (4). Currently, it is estimated that the health care costs associated with diabetes and 
GDM in the United States are \$278 billion and \$1.3 billion per year (5).

It is well recognized that women with prior GDM suffer higher risks of diabetes and prediabetes later in life (6). Studies of postpartum risk of diabetes and prediabetes in women with prior GDM suggest early lifestyle intervention to prevent the progression to diabetes or prediabetes in this high-risk population. However, the risks of postpartum diabetes in women with prior GDM might change at different conditions including different postpartum periods, maternal age, pre-pregnancy and postpartum BMI, ethnicity, diagnostic criteria and methods of GDM during pregnancy and diagnostic criteria and methods of postpartum diabetes. Until now, no previous studies have carried out an urban GDM universal screening survey by using World Health Organization (WHO)'s criteria and very few studies have assessed early postpartum diabetes and prediabetes by using a standard oral glucose tolerance test (OGTT) among women with and without a history of GDM. In the present analysis of the Tianjin GDM observational study, we aimed to investigate risks of early postpartum diabetes and prediabetes among women with and without a history of GDM.

\section{Subjects and methods}

\section{Tianjin GDM screening project}

Tianjin is the fourth largest city in China, only 30-min distance by train from Beijing. There are six central districts in Tianjin with about 4.3 million residents. In 1999, the Tianjin Women's and Children's Health Center launched an urban universal screening of GDM using WHO's criteria in all six central districts (2). The screening rate was reported to be $>91 \%$ between 1999 and 2008 (2). We used a 1-h glucose screening test with $50 \mathrm{~g}$ glucose load at 26-30 gestational weeks. If the 1-h glucose level was over $7.8 \mathrm{mmol} / \mathrm{L}$, another 2-h OGTT with $75 \mathrm{~g}$ glucose load would be performed at the Tianjin Women's and Children's Health Center. GDM diagnosis was made as per WHO criteria: a 75-g glucose 2-h OGTT result confirming either diabetes (fasting glucose $\geq 7 \mathrm{mmol} / \mathrm{L}$ or 2 -h glucose $\geq 11.1 \mathrm{mmol} / \mathrm{L}$ ) or impaired glucose tolerance (IGT) (2-h glucose $\geq 7.8$ and $<11.1 \mathrm{mmol} / \mathrm{L}$ ) (7).

\section{Study samples}

Totally 76325 women were screened from 2005 to 2009, among whom 4644 women were diagnosed as GDM and 71681 were free of GDM (Supplementary Fig. 1, see section on supplementary data given at the end of this article). We invited all 4644 GDM women to participate in the Tianjin Gestational Diabetes Mellitus Prevention Program (TGDMPP) $(8,9,10,11)$. Finally, 1263 women with GDM finished the baseline survey including a questionnaire and an OGTT between August 2009 and July 2011. Of these 1263 women with GDM, 83 were newly diagnosed diabetes according to the OGTT results and the rest 1180 women with GDM were randomized into an intervention group ( $n=586)$ and a 'usual care' control group $(n=594)$ in the TGDMPP. In parallel, we also enrolled in the Tianjin GDM observational study 705 non-GDM women and their children with birth dates and sex frequencymatched to the 594 children of GDM women in the 'usual care' group and 83 children of GDM women who were newly diagnosed diabetes at baseline survey. The study was approved by the Human Subjects Committee of the Tianjin Women's and Children's Health Center. All the participants provided written informed consent.

\section{Questionnaires and measurements}

All study participants filled in a questionnaire about their socio-demographics (age, marital status, education, income and occupation), history of GDM, family history (diabetes, coronary heart disease (CHD), stroke, cancer and hypertension), medical history (hypertension, diabetes and hypercholesterolemia), pregnancy outcomes (pre-pregnancy weight, weight gain in pregnancy and number of children), dietary habits (a self-administered food frequency questionnaire (FFQ) to measure the frequency and quantity of intake of 33 major food groups and beverages during the past year) (12), alcohol intake, smoking habits, passive smoking and physical activity (the frequency and duration of leisure-time and sedentary activities) at the postpartum baseline survey. They also completed the 3-day 24-h food records using methods for dietary record collections taught by a dietician. The performance of 3-day 24-h food records (12), the FFQ (12), and the above questionnaire on assessing physical activity $(13,14)$ have been validated in the China National Nutrition and Health Survey in 2002.

Body weight and height were measured using the standardized protocol by specially trained research doctors. BMI was calculated as the body weight in kilograms divided by the square of the height in meters. Waist circumference was measured at the horizontal level between the inferior costal margin and the iliac crest on the mid-axillary line with women in their standing position. Blood samples were collected in all participants after an overnight fast of 
at least $12 \mathrm{~h}$. Participants without a self-reported history of diabetes were given a standard 75-g glucose OGTT test. Plasma glucose was measured on an automatic analyzer (Toshiba TBA-120FR, Japan). Coefficient of variance was $4.42 \%$ for glucose.

\section{Definition of postpartum diabetes and prediabetes}

We used American Diabetes Association (ADA)'s criteria (15) for the diagnosis of diabetes and prediabetes. Diabetes was defined as fasting glucose $\geq 7.0 \mathrm{mmol} / \mathrm{L}$ and/or 2 -h glucose $\geq 11.1 \mathrm{mmol} / \mathrm{L}$. Prediabetes was defined as either impaired fasting glucose (fasting glucose $\geq 5.6 \mathrm{mmol} / \mathrm{L}$ and $<7.0 \mathrm{mmol} / \mathrm{L}$ ) or IGT (2-h glucose $\geq 7.8 \mathrm{mmol} / \mathrm{L}$ and $<11.1 \mathrm{mmol} / \mathrm{L})$. Those using antidiabetic drugs in the examination were also included into the type 2 diabetes cases. Until now, GDM women in both intervention and control groups in the TGDMPP finished baseline and subsequent annual OGTT during the 3-year follow-up visits. The present study included two different postpartum follow-up periods: early postpartum follow-up (both GDM and non-GDM women at baseline survey) and extended postpartum follow-up (GDM women in the interventional group and non-GDM women at baseline survey, and GDM women in the control group at the year 3 follow-up visit).

\section{Statistical analysis}

Standard $t$ test and chi-square test were used in the comparison of continuous variables and categorical variables between women with GDM and without GDM. Cox proportional hazard regression analysis was used to assess risks of postpartum diabetes and prediabetes between women with and without GDM. All analyses were adjusted for age (Model 1), and then for education, family income, family history of diabetes, smoking, passive smoking, alcohol drinking, leisure-time physical activity, sleeping time, dietary fiber, sweetened beverage drinking, energy intakes of fat, protein and carbohydrate (Model 2), and further for BMI (Model 3). $P<0.05$ was considered statistically significant. All statistical analyses were performed by IBM SPSS Statistics for Windows, version 24.0 (IBM Corp.).

\section{Results}

General characteristics of the study population at baseline were given in Table 1. Women with GDM were slightly older after delivery and younger at baseline survey. Their
BMI, HbA1C, fasting and 2-h glucose at baseline were higher. They were less educated and had less family income. In addition, they were less alcohol drinkers, less physically active and had more energy intakes as compared with those who were free of GDM.

During a mean follow-up of 3.53 years postpartum, there were 90 incident cases of diabetes and 599 incident cases of prediabetes. The incident rates of diabetes and prediabetes were 28.7/1000 person-years and 150.3/1000 person-years in GDM women, and 1.73/1000 personyears and 49.3/1000 person-years in non-GDM women, respectively. Multivariable-adjusted (age, education, family income, family history of diabetes, smoking, passive smoking, alcohol drinking, leisure-time physical activity, sleeping time, dietary fiber, sweetened beverage drinking, energy intakes of fat, protein and carbohydrate, and BMI - Model 3) hazard ratios among women with prior GDM, compared with those without GDM, were 76.1 (95\% CI: 23.6-246) for diabetes and 25.4 (95\% CI: 18.2-35.3) for prediabetes respectively (Fig. 1A, B and Table 2). When the mean follow-up extended to 4.40 years, there were 121 incident cases of diabetes and 616 incident cases of prediabetes. The incident rates of diabetes and prediabetes changed to $24.7 / 1000$ person-years and 98.1/1000 personyears in GDM women, and 1.73/1000 person-years and 49.3/1000 person-years in non-GDM women respectively. Women with prior GDM had a 13.0-fold multivariableadjusted risk (95\% CI: 5.54-30.6) for diabetes and 2.15fold risk (95\% CI: 1.76-2.62) for prediabetes compared with women without GDM (Fig. 1C, D and Table 2).

Multivariable-adjusted positive associations between GDM and the risks of postpartum diabetes and prediabetes were present in women aged younger and older than 30 years at delivery (Table 3). Similarly, the positive association was observed in both healthy weight women and overweight women. There were no statistically significant interactions of age, BMI and GDM status on the risks of postpartum diabetes and prediabetes (all $P$ for interaction $>0.25$ ).

\section{Discussion}

In this large GDM observational study, women with a history of GDM during pregnancy had a significantly increased risk of postpartum diabetes and prediabetes compared with women without GDM, and this risk was the highest at the first 3-4 years after delivery.

The prevalence of GDM is high among Asian women $(\sim 10 \%)$ compared with other racial/ethnic 
Table 1 Characteristics of women with and without gestational diabetes (GDM), Tianjin, China.

\begin{tabular}{l}
\hline \\
\hline No. of participants \\
Age at delivery (years) \\
Age at baseline (years) \\
Duration after delivery (years) \\
Weight gain during pregnancy (kg) \\
BMI (kg/m²) \\
Waist circumference (cm) \\
Fasting glucose (mmol/L) \\
2-hour glucose (mmol/L) \\
HbA1c (mmol/mol) \\
HbA1c (\%) \\
Education (\%) \\
$<13$ years \\
$13-16$ years \\
$\geq 16$ years \\
Income (\%) \\
$<5000$ yuan per month \\
$5000-8000$ yuan per month \\
$\geq 8000$ yuan per month \\
Family history of diabetes (\%) \\
Current smoking (\%) \\
Passive smoking (\%) \\
Current alcohol drinker (\%) \\
Leisure-time physical activity (\%) \\
0 min per day \\
$1-29$ min per day \\
$\geq 30$ min per day \\
Sleeping time (h/day) \\
Energy consumption (kcal/day) \\
Fiber (g/day) \\
Fat (\% energy) \\
Carbohydrate (\% energy) \\
Protein (\% energy) \\
Sweetened beverage drink (\%) \\
\hline
\end{tabular}

\begin{tabular}{c}
\hline Non-GDM \\
\hline 705 \\
$29.7 \pm 2.83$ \\
$35.4 \pm 2.95$ \\
$5.74 \pm 1.19$ \\
$18.3 \pm 6.67$ \\
$22.9 \pm 3.68$ \\
$75.8 \pm 8.26$ \\
$5.23 \pm 0.52$ \\
$6.14 \pm 1.41$ \\
$35 \pm 3$ \\
$5.3 \pm 0.2$ \\
10.4 \\
75.5 \\
14.2 \\
5.4 \\
15.5 \\
79.1 \\
27.1 \\
4.0 \\
55.2 \\
32.1 \\
61.7 \\
33.8 \\
4.5 \\
$7.48 \pm 0.95$ \\
$1627 \pm 381$ \\
$11.6 \pm 4.42$ \\
$31.1 \pm 5.65$ \\
$52.3 \pm 6.81$ \\
$16.6 \pm 2.62$ \\
77.9 \\
\\
\\
\\
\\
\end{tabular}

\begin{tabular}{c}
$\begin{array}{c}\text { GDM with early postpartum } \\
\text { follow-up }\end{array}$ \\
\hline 1263 \\
$30.1 \pm 3.50$ \\
$32.4 \pm 3.52$ \\
$2.29 \pm 0.88$ \\
$16.8 \pm 5.99$ \\
$24.2 \pm 3.93$ \\
$80.6 \pm 9.47$ \\
$5.38 \pm 0.97$ \\
$7.08 \pm 2.49$ \\
$38 \pm 8$ \\
$5.6 \pm 0.7$ \\
22.5 \\
70.1 \\
7.4 \\
27.5 \\
36.9 \\
35.6 \\
35.7 \\
2.0 \\
53.8 \\
21.8 \\
78.8 \\
19.1 \\
2.1 \\
$7.81 \pm 1.06$ \\
$1676 \pm 436$ \\
$12.2 \pm 4.75$ \\
$33.5 \pm 6.34$ \\
$49.5 \pm 7.30$ \\
$17.0 \pm 2.78$ \\
75.5 \\
\\
\\
\end{tabular}

\begin{tabular}{|c|c|}
\hline $\begin{array}{l}\text { GDM with extended postpartum } \\
\text { follow-up }\end{array}$ & $P$ value $^{b}$ \\
\hline \multicolumn{2}{|l|}{1263} \\
\hline $30.1 \pm 3.50$ & 0.008 \\
\hline $33.7 \pm 4.13$ & $<0.001$ \\
\hline $3.65 \pm 2.17$ & $<0.001$ \\
\hline $16.8 \pm 5.99$ & $<0.001$ \\
\hline $24.3 \pm 3.96$ & $<0.001$ \\
\hline $80.6 \pm 9.48$ & $<0.001$ \\
\hline $5.43 \pm 0.99$ & $<0.001$ \\
\hline $7.24 \pm 2.65$ & $<0.001$ \\
\hline $38 \pm 8$ & $<0.001$ \\
\hline \multirow{2}{*}{$5.6 \pm 0.7$} & $<0.001$ \\
\hline & $<0.001$ \\
\hline \multicolumn{2}{|l|}{22.5} \\
\hline \multicolumn{2}{|l|}{70.1} \\
\hline \multicolumn{2}{|l|}{7.4} \\
\hline \multirow{2}{*}{\multicolumn{2}{|c|}{$<0.001$}} \\
\hline & \\
\hline \multicolumn{2}{|l|}{36.9} \\
\hline \multicolumn{2}{|l|}{35.6} \\
\hline 35.7 & $<0.001$ \\
\hline 2.5 & 0.355 \\
\hline 58.0 & 0.572 \\
\hline \multirow[t]{2}{*}{26.9} & $<0.001$ \\
\hline & $<0.001$ \\
\hline \multicolumn{2}{|l|}{71.7} \\
\hline \multicolumn{2}{|l|}{25.3} \\
\hline \multicolumn{2}{|l|}{3.0} \\
\hline $7.70 \pm 1.02$ & $<0.01$ \\
\hline $1704 \pm 432$ & 0.01 \\
\hline $12.3 \pm 4.62$ & 0.01 \\
\hline $33.1 \pm 6.12$ & $<0.001$ \\
\hline $50.2 \pm 7.04$ & $<0.001$ \\
\hline $16.7 \pm 2.68$ & 0.006 \\
\hline 78.1 & 0.223 \\
\hline
\end{tabular}

aDietary intakes are assessed by 3-day 24-h food records; ${ }^{\text {b }} P$ values between non-GDM and GDM with early postpartum follow-up.

groups (5-8\%) in the United States $(16,17,18,19)$. In China, the prevalence of GDM has increased from $2.4 \%$ in 1999 to $8.2 \%$ in $2012(2,3)$, now similar to the level in US women ( 7\%) (4). Recent studies have found that Asian Americans with GDM are more likely to develop diabetes than other racial/ethnic women with GDM in the US (20) although Asian Americans generally have lower BMI and the prevalence of overweight/obesity than other racial/ethnic Americans $(21,22)$. However, very few studies investigated the postpartum risks of diabetes and prediabetes in Chinese women with a history of GDM. In a cohort of Chinese women living in Hong Kong, 9.0\% of women with a history of GDM developed type 2 diabetes after a median follow-up of 8 years (23).

It has been suggested that the risk of postpartum diabetes in women with prior GDM might change due to different races and ethnicities (24), different postpartum periods, maternal age, pre-pregnancy and postpartum BMI, diagnostic criteria of GDM during pregnancy and diagnostic criteria of postpartum diabetes. For example, Bao et al. reported a higher risk of postpartum diabetes among GDM women during up to 18 years of follow-up, especially among GDM women with higher baseline BMI and more weight gain after GDM development by a standard questionnaire (25). Since most of previous studies only used electronic medical records to identify symptomatic women at high risks of GDM and postpartum diabetes, these studies could have missed some asymptomatic cases of GDM and postpartum diabetes. No previous studies have reported the results based on an urban GDM universal screening survey by using the standard WHO's criteria, and very few studies have assessed early postpartum diabetes and prediabetes 

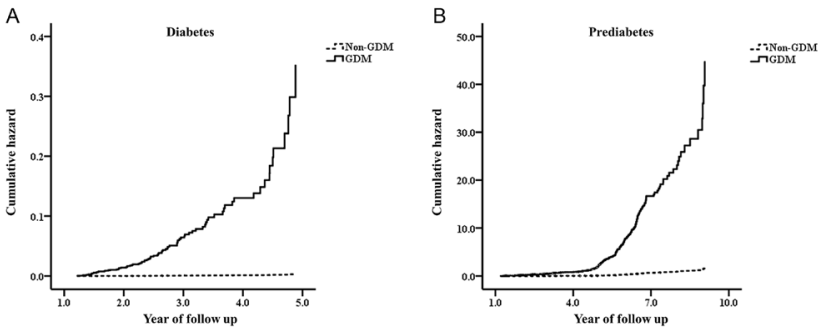

C
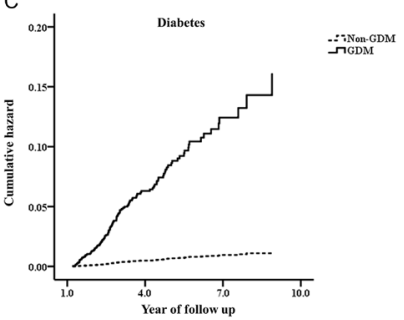

D

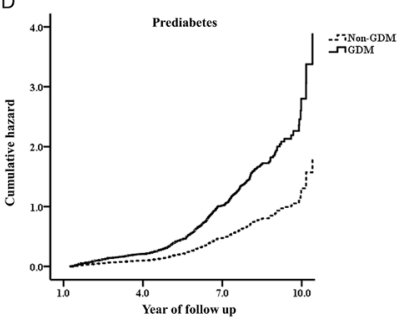

Figure 1

The cumulative incidence curve of type 2 diabetes ( $A$, early postpartum follow-up and C, extended postpartum follow-up) and prediabetes (B, early postpartum follow-up and $D$, extended postpartum follow-up) among women with and without GDM. All analyses were adjusted for age, education, family income, family history of diabetes, current smoking, passive smoking, current alcohol drinking, leisure-time physical activity, sleeping time, dietary fiber, sweetened beverage drinking, energy intakes of fat, protein, and carbohydrate and BMI. GDM, gestational diabetes mellitus.

by using a standard OGTT among women with and without a history of GDM. Tianjin GDM observational study was the only study with a large sample size including 1263 women with a history of GDM and 705 women without GDM from 76325 women who participated in the whole population's GDM universal screening survey by using the WHO's criteria. All 1263 women with prior GDM and 705 women with GDM had a standard OGTT at 1-9 years postpartum to identify postpartum diabetes and prediabetes using the ADA's criteria. In addition, about $95 \%$ of pregnancy women in the Tianjin urban area finished a fasting glucose examination during the first trimester (26). Women with diabetes before pregnancy or with asymptomatic diabetes during the early pregnancy were excluded in the present study. Thus, the present study provided a comprehensive and accurate estimation of GDM with postpartum diabetes and prediabetes.

Two early reviews have reported that women with prior GDM have a seven-fold higher risk of postpartum diabetes compared with women without GDM $(6,27)$. A recent review has also indicated that the adjusted odds ratios of diabetes among GDM women compared

with non-GDM women at <3, 3-6 and 6-10 years after delivery were 5.37, 16.55 and 8.20, respectively (28). The present study found that women with prior GDM had a 76.1-fold risk for diabetes and a 25.4-fold risk for prediabetes compared with women without GDM at a mean follow-up of 3.53 years postpartum. When the mean follow-up extended to 4.40 years, women with prior GDM had a 13.0-fold risk for diabetes and a 2.15fold risk for prediabetes compared with women without GDM. The present study demonstrated that women with prior GDM had the highest risk of postpartum diabetes at the first 3-4 years after delivery compared with women without GDM, which was similar to the trend from one recent meta-analysis that the risk of postpartum diabetes among women with GDM increased to a peak at the first 3-6 years and then attenuated (28). Explanations might include more pronounced beta cell defect and susceptible genetic variants among women with early onset of postpartum diabetes (29). Several studies have confirmed that antepartum characteristics might also contribute to the onset of postpartum diabetes $(30,31)$. However, in our cohort, there were no significant differences in antepartum or postpartum BMI, weight gain during pregnancy and the GDM women's insulin treatment during pregnancy among women with early onset of postpartum diabetes compared with women with late onset of postpartum diabetes. Whether this risk trend resulted from other factors remains unknown and needs further studies. Thus, we implied that women with prior GDM represents a high risk population that should be targeted for early postpartum lifestyle intervention in order to prevent or delay the onset of type 2 diabetes. TGDMPP is an ongoing trial to evaluate the effect of early postpartum lifestyle intervention on the progress of diabetes.

In subgroup analyses of the present study, overweight women with prior GDM seemed to show a much higher risk for postpartum diabetes than normal weight women with prior GDM compared with overweight and normal weight women without GDM, which was similar with the results in other studies $(32,33)$. Overweight is a definite risk factor for diabetes and prediabetes. Thus, early postpartum lifestyle intervention on weight loss is strongly recommended to prevent or delay type 2 diabetes and prediabetes among overweight women with a history of GDM. Meanwhile, we also noticed that women with GDM who were younger than age 30 years at delivery tended to have higher risks for postpartum diabetes and prediabetes than women with prior GDM who were older than age 30 years at delivery, which should be taken more attention. The early postpartum 2-h OGTT might 
Table 2 Hazard ratio of diabetes and prediabetes among women with and without GDM at different follow-up period. Model 1 was adjusted for age; Model 2 was adjusted for age, education, family income, family history of diabetes, current smoking, passive smoking, current alcohol drinking, leisure-time physical activity, sleeping time, energy consumption, fiber, fat, protein and carbohydrate consumption, and sweetened beverage drinking; Model 3 was adjusted for factors in Model 2 and also BMI.

\begin{tabular}{|c|c|c|c|c|}
\hline & \multicolumn{2}{|c|}{ Diabetes } & \multicolumn{2}{|c|}{ Prediabetes $^{\mathrm{a}}$} \\
\hline & Non-GDM & GDM & Non-GDM & GDM \\
\hline \multicolumn{5}{|c|}{ GDM women with early postpartum follow-up (mean 3.53 years) } \\
\hline No. of participants & 705 & 1263 & 698 & 1180 \\
\hline No. of cases & 7 & 83 & 198 & 401 \\
\hline Pearson-years & 4052 & 2886 & 4014 & 2668 \\
\hline \multicolumn{5}{|c|}{ Multiple adjusted hazard ratios } \\
\hline Model 1 & 1 & $113(37.3-346)$ & 1 & $29.0(21.2-39.5)$ \\
\hline Model 2 & 1 & $91.0(28.2-294)$ & 1 & $27.0(19.5-37.5)$ \\
\hline Model 3 & 1 & $76.1(23.6-246)$ & 1 & $25.4(18.2-35.3)$ \\
\hline \multicolumn{5}{|c|}{ GDM women with extended postpartum follow-up (mean 4.40 years) } \\
\hline No. of participants & 705 & 1263 & 698 & 1149 \\
\hline No. of cases & 7 & 114 & 198 & 418 \\
\hline Pearson-years & 4052 & 4609 & 4014 & 4262 \\
\hline \multicolumn{5}{|c|}{ Multiple adjusted hazard ratios } \\
\hline Model 1 & 1 & $17.0(7.92-36.6)$ & 1 & $2.20(1.85-2.62)$ \\
\hline Model 2 & 1 & $15.6(6.62-36.7)$ & 1 & $2.30(1.89-2.80)$ \\
\hline Model 3 & 1 & $13.0(5.54-30.6)$ & 1 & $2.15(1.76-2.62)$ \\
\hline
\end{tabular}

help identify asymptomatic women with diabetes and prediabetes at a younger age, which could partly explain the higher risk of women at a younger age. In addition, GDM women who were younger at delivery in our cohort were more exposed to positive family history of diabetes. These women may be of higher genetic susceptibility to glucose intolerance as they developed GDM even at a younger age. Patients diagnosed as type 2 diabetes at a younger age usually have much higher risks of macro- (34) and microvascular diseases (35) than those developing type 2 diabetes later. The findings of the present study will greatly promote favorable changes in the Public Health Policy regarding prevention of the progress of diabetes among women with a history of GDM.

A unique advantage of the present study is that diagnoses of GDM at 26-30 gestational weeks were based on the whole population's GDM universal screening by using the WHO's criteria after a $2 \mathrm{~h} 75 \mathrm{~g}$ OGTT. The diagnosis of postpartum diabetes and prediabetes was based on the ADA's criteria after a $2 \mathrm{~h} 75 \mathrm{~g}$ OGTT. Our

Table 3 Hazard ratio of diabetes and prediabetes among women with and without GDM by various subgroups.

\begin{tabular}{|c|c|c|c|c|}
\hline & \multicolumn{2}{|c|}{ Diabetes } & \multicolumn{2}{|c|}{ Prediabetes $^{\mathrm{a}}$} \\
\hline & Non-GDM & GDM & Non-GDM & GDM \\
\hline \multicolumn{5}{|c|}{ Women with early postpartum follow-up (mean 3.53 years) } \\
\hline \multicolumn{5}{|c|}{ BMI $\left(\mathrm{kg} / \mathrm{m}^{2}\right)^{\mathrm{c}}$} \\
\hline$<25$ & 1 & $50.2(13.1-193)$ & 1 & $26.8(17.8-40.5)$ \\
\hline$\geq 25$ & 1 & $164(18.3-1464)$ & 1 & $25.3(14.3-44.8)$ \\
\hline \multicolumn{5}{|c|}{ Age at delivery groups (years) ${ }^{c}$} \\
\hline$<30$ & 1 & $132(22.6-774)$ & 1 & $28.6(18.2-44.6)$ \\
\hline$\geq 30$ & 1 & $60.0(9.93-363)$ & 1 & $29.3(16.8-50.9)$ \\
\hline \multicolumn{5}{|c|}{ Women with extended postpartum follow-up (mean 4.40 years) ${ }^{b}$} \\
\hline \multicolumn{5}{|c|}{ BMI $\left(\mathrm{kg} / \mathrm{m}^{2}\right)^{\mathrm{c}}$} \\
\hline$<25$ & 1 & $8.45(2.67-26.8)$ & 1 & $2.47(1.92-3.18)$ \\
\hline$\geq 25$ & 1 & $22.0(5.26-91.9)$ & 1 & $1.78(1.29-2.46)$ \\
\hline \multicolumn{5}{|c|}{ Age at delivery groups (years) ${ }^{c}$} \\
\hline$<30$ & 1 & $19.1(5.76-63.3)$ & 1 & $2.20(1.69-2.85)$ \\
\hline$\geq 30$ & 1 & $7.91(2.36-26.5)$ & 1 & $2.14(1.57-2.91)$ \\
\hline
\end{tabular}


study is the only study to provide a comprehensive and accurate estimation of GDM with postpartum diabetes and prediabetes. Another important strength of our study is the large sample size of both women with and without a history of GDM. One limitation of this study is the return rate of the initial invitation with only $27 \%$ of all GDM women. Although there were no differences in age, 2-h glucose, fasting glucose, the prevalence of IGT and diabetes at 26-30 gestational weeks and OGTT tests between those returned and those not returned, whether other differences between groups existed cannot be verified. Second, we have no data on 1-h glucose and 3-h glucose during pregnancy since we used the WHO's diagnostic criteria for GDM. Finally, due to the one-child policy from 1979 to 2015 (36), the birth rates were lower in China than in other countries, which may influence the results. It has been considered that increasing numbers of parity were associated with an increasing risk of type 2 diabetes (37). However, since only $2.44 \%$ of the women included in the Tianjin GDM screening project have a history of more than one parity (2), parity bias is not considered in the present study.

In conclusion, we reported a substantially increased risk of postpartum diabetes and prediabetes in women with prior GDM compared with those without GDM, and this risk was the highest at the first 3-4 years after delivery. An early postpartum lifestyle intervention might prevent and delay diabetes and prediabetes risk among women with a history of GDM.

\section{Supplementary data}

This is linked to the online version of the paper at https://doi.org/10.1530/ EJE-18-0130.

\section{Declaration of interest}

The authors declare that there is no conflict of interest that could be perceived as prejudicing the impartiality of this study.

\section{Funding}

This study is supported by the grant from European Foundation for the Study of Diabetes (EFSD)/Chinese Diabetes Society (CDS)/Lilly programme for Collaborative Research between China and Europe, Tianjin Women's and Children's Health Center and Tianjin Public Health Bureau. Dr Hu was partly supported by the grant from the National Institute of Diabetes and Digestive and Kidney Diseases (R01DK100790) and the National Institute of General Medical Sciences (U54GM104940) of the National Institutes of Health. Dr Cuilin Zhang is supported by the Intramural Research Program of Eunice Kennedy Shriver National Institute of Child Health and Human Development, National Institutes of Health. The funding sources had no role in the design and conduct of the study, collection, management, analysis and interpretation of the data, preparation, review or approval of the manuscript and decision to submit the manuscript for publication.
Author contribution statement

Y S, P W and G H designed the study, acquired data, performed statistical analyses and drafted the manuscript. $\mathrm{H}$ T and $\mathrm{J} \mathrm{T}$ designed the study and critically revised the manuscript for important intellectual content. $L$ W, S Z, H L, W L, N L, W L, J L and J W acquired data, performed statistical analyses and critically revised the manuscript for important intellectual content. C Z, X Y and $Z Y$ critically revised the manuscript for important intellectual content. G $\mathrm{H}$ is the guarantor of this work and, as such, had full access to all the data in the study and takes responsibility for the integrity of the data and the accuracy of the data analysis.

\section{Acknowledgements}

The authors would like to appreciate all families for participating in the Tianjin Gestational Diabetes Mellitus Prevention Program.

\section{References}

1 Wang L, Gao P, Zhang M, Huang Z, Zhang D, Deng Q, Li Y, Zhao Z, Qin X, Jin D et al. Prevalence and ethnic pattern of diabetes and prediabetes in China in 2013. JAMA 2017317 2515-2523. (https:// doi.org/10.1001/jama.2017.7596)

2 Zhang F, Dong L, Zhang C, Li B, Wen J, Gao W, Sun S, Lv F, Tian H, Tuomilehto $\mathrm{J}$ et al. Increasing prevalence of gestational diabetes mellitus in Chinese women from 1999 to 2008. Diabetic Medicine 201128 652-657. (https://doi.org/10.1111/j.14645491.2010.03205.x)

3 Leng J, Shao P, Zhang C, Tian H, Zhang F, Zhang S, Dong L, Li L, Yu Z, Chan JC et al. Prevalence of gestational diabetes mellitus and its risk factors in chinese pregnant women: a prospective populationbased study in Tianjin, China. PLoS ONE 201510 e0121029. (https:// doi.org/10.1371/journal.pone.0121029)

4 American Diabetes Association. Gestational diabetes mellitus. Diabetes Care 200326 (Supplement 1) S103-S105. (https://doi. org/10.2337/diacare.26.2007.S103)

5 Dall TM, Yang W, Halder P, Pang B, Massoudi M, Wintfeld N, Semilla AP, Franz J \& Hogan PF. The economic burden of elevated blood glucose levels in 2012: diagnosed and undiagnosed diabetes, gestational diabetes mellitus, and prediabetes. Diabetes Care 201437 3172-3179. (https://doi.org/10.2337/dc14-1036)

6 Bellamy L, Casas JP, Hingorani AD \& Williams D. Type 2 diabetes mellitus after gestational diabetes: a systematic review and metaanalysis. Lancet 2009373 1773-1779. (https://doi.org/10.1016/ S0140-6736(09)60731-5)

7 WHO Consultation. Definition, Diagnosis and Classification of Diabetes Mellitus and its Complications. Part 1: Diagnosis and Classification of Diabetes Mellitus. Geneva: World Health Organisation, 1999.

8 Hu G, Tian H, Zhang F, Liu H, Zhang C, Zhang S, Wang L, Liu G, Yu Z, Yang X et al. Tianjin Gestational Diabetes Mellitus Prevention Program: study design, methods, and 1-year interim report on the feasibility of lifestyle intervention program. Diabetes Research and Clinical Practice 201298 508-517. (https://doi.org/10.1016/j. diabres.2012.09.015)

9 Liu H, Zhang C, Zhang S, Wang L, Leng J, Liu D, Fang H, Li W, Yu Z, Yang X et al. Joint effects of pre-pregnancy body mass index and weight change on postpartum diabetes risk among gestational diabetes women. Obesity 201422 1560-1567. (https://doi. org/10.1002/oby.20722)

10 Liu H, Zhang S, Wang L, Leng J, Li W, Li N, Li M, Qiao Y, Tian H, Tuomilehto J et al. Fasting and 2-hour plasma glucose, and HbA1c in pregnancy and the postpartum risk of diabetes among Chinese women with gestational diabetes. Diabetes Research and Clinical Practice 2016112 30-36. (https://doi.org/10.1016/j. diabres.2015.11.007) 
11 Li W, Zhang S, Liu H, Wang L, Zhang C, Leng J, Yu Z, Yang X, Tian H $\&$ Hu G. Different associations of diabetes with beta-cell dysfunction and insulin resistance among obese and nonobese Chinese women with prior gestational diabetes mellitus. Diabetes Care 201437 2533-2539. (https://doi.org/10.2337/dc14-0573)

12 Li YP, He YN, Zhai FY, Yang XG, Hu XQ, Zhao WH \& Ma GS. Comparison of assessment of food intakes by using 3 dietary survey methods. Zhonghua Yu Fang Yi Xue Za Zhi 200640 273-280. (https:// doi.org/10.3760/j:issn:0253-9624.2006.04.014)

13 Ma G, Luan D, Liu A, Li Y, Cui Z, Hu X \& Yang X. The analysis and evaluation of a physical activity questionnaire of Chinese employed population. Ying Yang Xue Bao 200729 217-221. (https://doi. org/10.3321/j.issn:0512-7955.2007.03.007)

14 Ma G, Luan D, Li Y, Liu A, Hu X, Cui Z, Zhai F \& Yang X. Physical activity level and its association with metabolic syndrome among an employed population in China. Obesity Review 20089 (Supplement 1) 113-118. (https://doi.org/10.1111/j.1467-789X.2007.00451.x)

15 American Diabetes Association. 2. Classification and diagnosis of diabetes: standards of medical care in diabetes-2018. Diabetes Care 201841 S13-S27. (https://doi.org/10.2337/dc18-S002)

16 Chu SY, Abe K, Hall LR, Kim SY, Njoroge T \& Qin C. Gestational diabetes mellitus: all Asians are not alike. Preventive Medicine 200949 265-268. (https://doi.org/10.1016/j.ypmed.2009.07.001)

17 Ferrara A, Kahn HS, Quesenberry CP, Riley C \& Hedderson MM. An increase in the incidence of gestational diabetes mellitus: Northern California, 1991-2000. Obstetrics and Gynecology 2004103 526-533. (https://doi.org/10.1097/01.AOG.0000113623.18286.20)

18 Dabelea D, Snell-Bergeon JK, Hartsfield CL, Bischoff KJ, Hamman RF $\&$ McDuffie RS. Increasing prevalence of gestational diabetes mellitus (GDM) over time and by birth cohort: Kaiser Permanente of Colorado GDM Screening Program. Diabetes Care 200528 579-584. (https://doi.org/10.2337/diacare.28.3.579)

19 Wang Y, Chen L, Xiao K, Horswell R, Besse J, Johnson J, Ryan DH $\& \mathrm{Hu}$ G. Increasing incidence of gestational diabetes mellitus in louisiana, 1997-2009. Journal of Women's Health 201221 319-325. (https://doi.org/10.1089/jwh.2011.2838)

20 Wang Y, Chen L, Horswell R, Xiao K, Besse J, Johnson J, Ryan DH \& Hu G. Racial differences in the association between gestational diabetes mellitus and risk of type 2 diabetes. Journal of Women's Health 201221 628-633. (https://doi.org/10.1089/ jwh.2011.3318)

21 Oza-Frank R, Ali MK, Vaccarino V \& Narayan KM. Asian Americans: diabetes prevalence across U.S. and World Health Organization weight classifications. Diabetes Care 200932 1644-1646. (https://doi. org/10.2337/dc09-0573)

22 Shai I, Jiang R, Manson JE, Stampfer MJ, Willett WC, Colditz GA \& $\mathrm{Hu}$ FB. Ethnicity, obesity, and risk of type 2 diabetes in women: a 20-year follow-up study. Diabetes Care 200629 1585-1590. (https:// doi.org/10.2337/dc06-0057)

23 Tam WH, Yang XL, Chan JC, Ko GT, Tong PC, Ma RC, Cockram CS, Sahota D \& Rogers MS. Progression to impaired glucose regulation, diabetes and metabolic syndrome in Chinese women with a past history of gestational diabetes. Diabetes/Metabolism Research and Reviews 200723 485-489. (https://doi.org/10.1002/dmrr.741)

24 Xiang AH, Li BH, Black MH, Sacks DA, Buchanan TA, Jacobsen SJ \& Lawrence JM. Racial and ethnic disparities in diabetes risk after gestational diabetes mellitus. Diabetologia 201154 3016-3021. (https://doi.org/10.1007/s00125-011-2330-2)
25 Bao W, Yeung E, Tobias DK, Hu FB, Vaag AA, Chavarro JE, Mills JL, Grunnet LG, Bowers K, Ley SH et al. Long-term risk of type 2 diabetes mellitus in relation to BMI and weight change among women with a history of gestational diabetes mellitus: a prospective cohort study. Diabetologia 201558 1212-1219. (https://doi.org/10.1007/s00125015-3537-4)

26 Dong L, Liu E, Guo J, Pan L, Li B, Leng J, Zhang C, Zhang Y, Li N \& $\mathrm{Hu}$ G. Relationship between maternal fasting glucose levels at 4-12 gestational weeks and offspring growth and development in early infancy. Diabetes Research and Clinical Practice 2013102 210-217. (https://doi.org/10.1016/j.diabres.2013.10.017)

27 Kim C, Newton KM \& Knopp RH. Gestational diabetes and the incidence of type 2 diabetes: a systematic review. Diabetes Care 2002 25 1862-1868. (https://doi.org/10.2337/diacare.25.10.1862)

28 Song C, Lyu Y, Li C, Liu P, Li J, Ma RC \& Yang X. Long-term risk of diabetes in women at varying durations after gestational diabetes: a systematic review and meta-analysis with more than 2 million women. Obesity Reviews 201819 421-429. (https://doi.org/10.1111/ obr.12645)

29 Kwak SH, Choi SH, Jung HS, Cho YM, Lim S, Cho NH, Kim SY, Park KS \& Jang HC. Clinical and genetic risk factors for type 2 diabetes at early or late post partum after gestational diabetes mellitus. Journal of Clinical Endocrinology and Metabolism 201398 E744-E752. (https://doi.org/10.1210/jc.2012-3324)

30 Jang HC. Gestational diabetes in Korea: incidence and risk factors of diabetes in women with previous gestational diabetes. Diabetes and Metabolism Journal 201135 1-7. (https://doi.org/10.4093/ dmj.2011.35.1.1)

31 Buchanan TA, Xiang A, Kjos SL, Lee WP, Trigo E, Nader I, Bergner EA, Palmer JP \& Peters RK. Gestational diabetes: antepartum characteristics that predict postpartum glucose intolerance and type 2 diabetes in Latino women. Diabetes 199847 1302-1310. (https:// doi.org/10.2337/diab.47.8.1302)

32 Cianni GD, Ghio A, Resi V \& Volpe L. Gestational diabetes mellitus: an opportunity to prevent type 2 diabetes and cardiovascular disease in young women. Women's Health 20106 97-105. (https://doi. org/10.2217/whe.09.76)

33 Lee AJ, Hiscock RJ, Wein P, Walker SP \& Permezel M. Gestational diabetes mellitus: clinical predictors and long-term risk of developing type 2 diabetes: a retrospective cohort study using survival analysis. Diabetes Care 200730 878-883. (https://doi.org/10.2337/dc06-1816)

34 Huo X, Gao L, Guo L, Xu W, Wang W, Zhi X, Li L, Ren Y, Qi X, Sun Z et al. Risk of non-fatal cardiovascular diseases in early-onset versus late-onset type 2 diabetes in China: a cross-sectional study. Lancet Diabetes and Endocrinology 20164 115-124. (https://doi.org/10.1016/ S2213-8587(15)00508-2)

35 Li L, Ji L, Guo X, Ji Q, Gu W, Zhi X, Li X, Kuang H, Su B, Yan J et al. Prevalence of microvascular diseases among tertiary care Chinese with early versus late onset of type 2 diabetes. Journal of Diabetes and Its Complications 201529 32-37. (https://doi.org/10.1016/j. jdiacomp.2014.08.010)

36 Zeng Y \& Hesketh T. The effects of China's universal two-child policy. Lancet 2016388 1930-1938. (https://doi.org/10.1016/S01406736(16)31405-2)

37 Naver KV, Lundbye-Christensen S, Gorst-Rasmussen A, Nilas L, Secher NJ, Rasmussen S \& Ovesen P. Parity and risk of diabetes in a Danish nationwide birth cohort. Diabetic Medicine 201128 43-47. (https://doi.org/10.1111/j.1464-5491.2010.03169.x)

Received 12 February 2018

Revised version received 30 April 2018

Accepted 9 May 2018 